\title{
Gluino polarization at the LHC
}

\author{
M. Krämer, ${ }^{1}$ E. Popenda, ${ }^{1}$ M. Spira, ${ }^{2}$ and P. M. Zerwas ${ }^{3,1}$ \\ ${ }^{1}$ Institut für Theoretische Physik E, RWTH Aachen University, D-52074 Aachen, Germany \\ ${ }^{2}$ Paul Scherrer Institut, CH-5232 Villigen PSI, Switzerland \\ ${ }^{3}$ Deutsches Elektronen-Synchrotron DESY, D-22603 Hamburg, Germany
}

(Received 4 March 2009; published 1 September 2009)

\begin{abstract}
Gluinos are produced pairwise at the LHC in quark-antiquark and gluon-gluon collisions: $q \bar{q}, g g \rightarrow$ $\tilde{g} \tilde{g}$. While the individual polarization of gluinos vanishes in the limit in which the small mass difference between $L$ and $R$ squarks of the first two generations is neglected, nonzero spin-spin correlations are predicted within gluino pairs. If the squark/quark charges in Majorana gluino decays are tagged, the spin correlations have an impact on the energy and angular distributions in reconstructed final states. On the other hand, the gluino polarization in single gluino production in the supersymmetric Compton process $g q \rightarrow \tilde{g} \tilde{q}_{R, L}$ is predicted to be nonzero, and the polarization affects the final-state distributions in superCompton events.
\end{abstract}

DOI: 10.1103/PhysRevD.80.055002

PACS numbers: $12.60 . J v$

\section{INTRODUCTION}

Gluinos in the minimal supersymmetric standard model (MSSM) [1-3] can be produced copiously at the LHC, cf. Refs. [4,5]; gluino pairs in quark-antiquark and gluongluon collisions, Figs. 1(a) and 1(b),

$$
\begin{aligned}
& q \bar{q} \longrightarrow \tilde{g} \tilde{g}, \\
& g g \longrightarrow \tilde{g} \tilde{g},
\end{aligned}
$$

and single gluinos in association with squarks in the superCompton process, Fig. 1(c),

$$
q g \rightarrow \tilde{q} \tilde{g} .
$$

After the gluinos decay, the final-state energy and angular distributions will in general depend on the degree of polarization with which the gluinos are generated. ${ }^{1}$ Thus the distribution of experimentally reconstructed events is affected by the polarization of the gluinos.

However, polarization effects are to a large extent expunged by the Majorana character of the particles, in particular, when parton charges are not measured. For example, jet angular distributions in $^{2}$

$$
\tilde{g} \rightarrow q_{R, L} \tilde{q}_{R, L}^{*} \quad \text { and } \quad \tilde{g} \rightarrow \bar{q}_{L, R} \tilde{q}_{R, L}
$$

follow, mutually, the $\left[1 \pm \cos \theta_{*}\right]$ law with regard to the gluino spin vector so that the sum of the spin-dependent terms vanishes, as a result of $C P$ invariance if the $q, \tilde{q}$ charges are not analyzed. (For the sake of simplicity we will restrict ourselves to the class of SPS1a/a'-type scenarios [7] in which the gluino decay patterns are restricted

\footnotetext{
${ }^{1}$ Spin measurements of supersymmetric particles sui generis at the LHC are widely discussed in the literature; for a sample of methods that address the impact of spin on differential distributions see Ref. [6].

${ }^{2}$ The indices $R, L$ denote the \pm helicities of quarks and antiquarks.
}

to 2-body decays rather than involving more complex 3body decays which include virtual squarks for the opposite ratio of masses.)

If gluinos, on the other hand, were Dirac particles $\tilde{g}_{D}$ as may be formalized in $N=2$ hybrid models [8-11], the conservation of the Dirac charge $D$, with $D=+1$ for $\tilde{g}_{D}$, $\tilde{q}_{R}$ and -1 for $\tilde{g}_{D}^{c}, \tilde{q}_{L}[8]$, allows only the production of
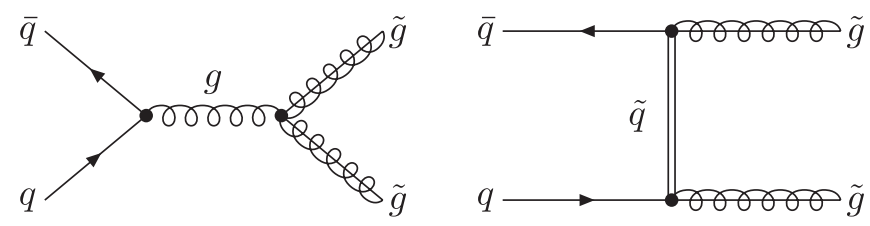

(a)
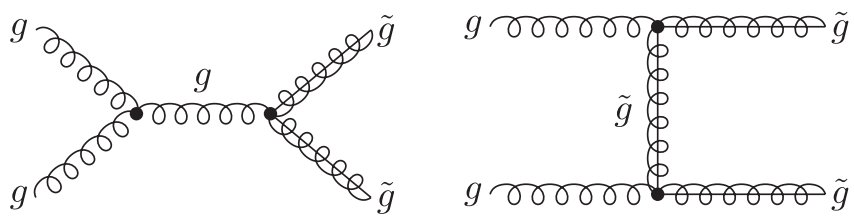

(b)
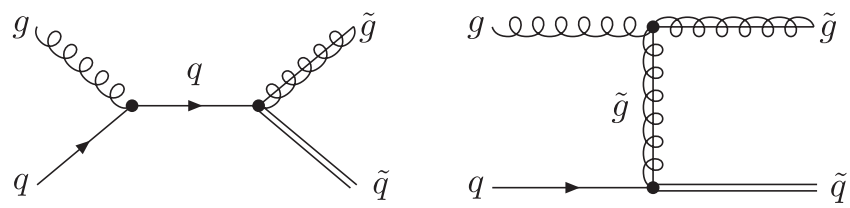

(c)

FIG. 1. Feynman diagrams for gluino production in quark annihilation (a), gluon fusion (b), and in the super-Compton process (c). 
specific pairs of supersymmetric particles and antiparticles: $\tilde{g}_{D} \tilde{g}_{D}^{c} ; \tilde{g}_{D} \tilde{q}_{L}, \tilde{g}_{D}^{c} \tilde{q}_{R}$ etc., followed by specific decay patterns: $\tilde{g}_{D} \rightarrow q_{L} \tilde{q}_{L}^{*}$ but not $\tilde{g}_{D} \rightarrow \bar{q}_{R} \tilde{q}_{L}$, and crosswise for $R$ squarks/antisquarks. This gives rise to stringent restrictions on polarization and spin-correlation effects.

This report should serve a twofold purpose. First, it will be analyzed in which processes gluino polarization may affect final-state distributions, and the mechanisms will be discussed which reduce such effects when, in particular, charges are summed in the final state. Second, the size of the primary spin effects will be illustrated, at the purely theoretical level, by studying jet-jet invariant mass distributions. Not presenting prescriptions which are systematically optimized for isolating any spin effects experimentally, the paper should rather point out which spin effects may eventually propagate into final states exploited for experimental high-precision analyses of supersymmetry processes at the LHC. Though their actual impact will largely depend on the experimental methods used in practice, the size of the effects must be controlled nevertheless. This paper should provide the theoretical basis for such analyses.

(1) Gluino pairs: The masses of $L$ and $R$ squarks of the first two generations are generally close to each other, although large mass differences are not excluded by any fundamental principle. For the SPS1a/a' benchmark point for instance, the difference over the sum of the two $L / R$ masses is just $2 \%$. Single gluino polarization is therefore constrained to $\mathcal{P} \sim\left[\tilde{m}_{L}-\tilde{m}_{R}\right] 2 / s$ at the percent level or less. (For details see Ref. [12].) Neglecting the small mass differences, the super-QCD action of the first two generations becomes invariant under $P$ transformation combined with the exchange of $\tilde{q}_{L} \leftrightarrow \tilde{q}_{R}$. As a result, the single polarization of gluinos vanishes in the pair-production processes (1.1) and (1.2) in this approximation. However, spin correlations within the gluino pair are nontrivial.

As argued before, the sum of polarization asymmetries adds up to zero in $C P$-invariant theories, if the charges are not measured in the gluino decay modes. Hence, the jets which originate in equal shares from quarks and antiquarks in (1.4) are isotropically distributed. The distribution in the scaled jet-jet mass, $m=M_{\mathrm{jj}} / \sqrt{s}$ in the decay $\tilde{g} \tilde{g} \rightarrow q q^{\prime}+$ $X$ is then moderately soft,

$$
\text { isotropic: } \sigma^{-1} d \sigma / d m^{2}=\log m^{-2}
$$

for asymptotic energies $s \gg M_{\tilde{g}}^{2} \gg M_{\tilde{q}}^{2}[6,13]$. However, charges can be tagged in $\tilde{u}_{L}, \tilde{d}_{L}$ decays to charginos, $\tilde{q}_{L} \rightarrow$ $q \tilde{\chi}_{1}^{ \pm} \rightarrow q l^{ \pm} \nu_{l} \tilde{\chi}_{1}^{0}$. This is applicable in scenarios in which $\tilde{\chi}_{2}^{0}$, for instance, is not a pure wino or bino state, leading to different total $\tilde{u}_{L}$ and $\tilde{d}_{L}^{*}$ widths and, as a result, unequal branching ratios. In such scenarios polarization effects do not completely cancel among the decay chains $\tilde{g} \rightarrow$ $\bar{u}_{R} \tilde{u}_{L} \rightarrow \bar{u}_{R} d \tilde{\chi}_{1}^{+}$and $\tilde{g} \rightarrow d_{L} \tilde{d}_{L}^{*} \rightarrow d_{L} \bar{u} \tilde{\chi}_{1}^{+}$, which result in identical final-state charges, but have opposite polarization signatures. Tagging of top and bottom charges in the

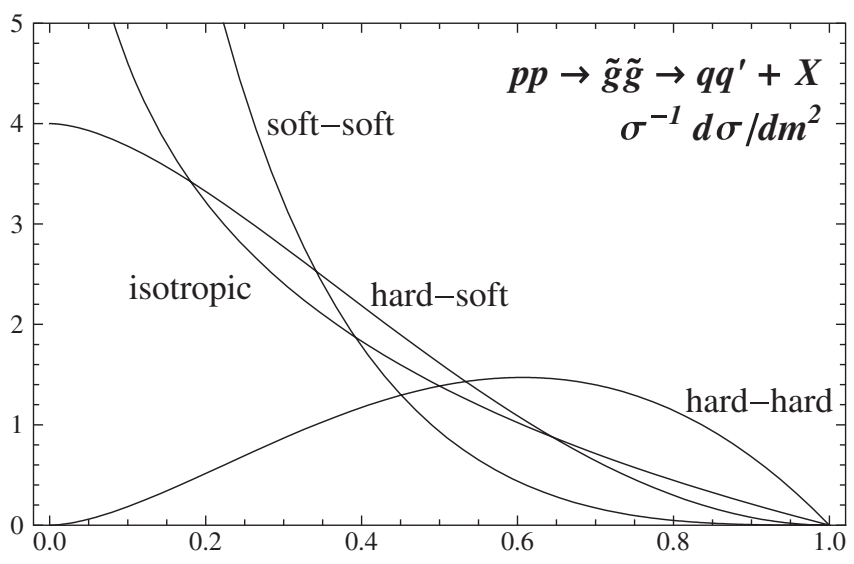

(a)

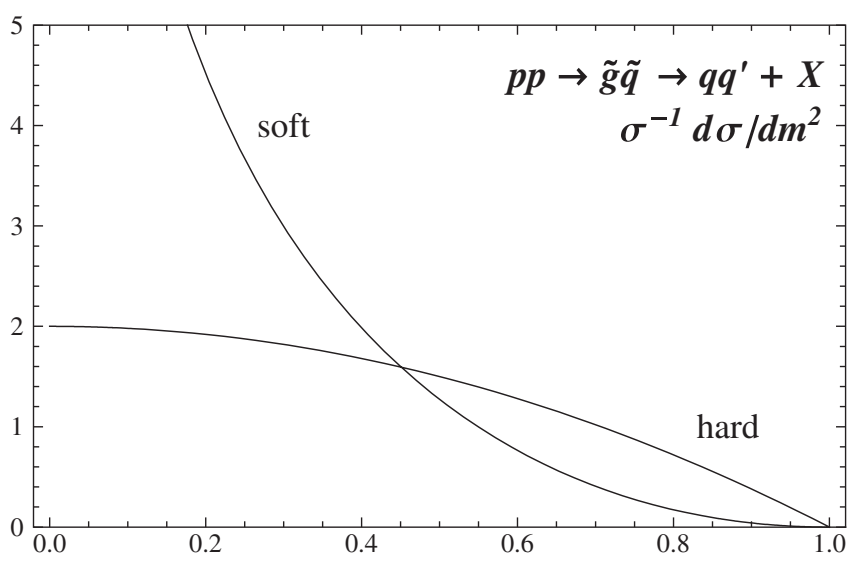

(b)

$m$

FIG. 2. Invariant near-jet-jet mass distributions in the gluinopair production processes (a) and the super-Compton process (b) for various combinations of hard and soft fragmentation $\tilde{g} \rightarrow q$.

third generation can be exploited in any case, though spin effects will be diluted by $4-b$ tagging through (nonleptonic) $b$ identification and jet-charge measurement. In the asymptotic limit in which gluino fragmentation to quarks (plus accompanying squarks) is either hard or soft, i.e. $\sim 2 z$ or $2(1-z)$ for quarks emitted preferentially parallel or antiparallel to the gluino spin, various configurations can be realized for the invariant mass distributions of the nearjet pairs ${ }^{3}$ in $\tilde{g} \tilde{g} \rightarrow q q^{\prime}+X$ final states [13]:

hard-hard: $\sigma^{-1} d \sigma / d m^{2}=4 m^{2} \log m^{-2}$

hard-soft:

$$
\begin{aligned}
& =4\left[\left(1-m^{2}\right)-m^{2} \log m^{-2}\right] \\
& =4\left[\left(1+m^{2}\right) \log m^{-2}-2\left(1-m^{2}\right)\right] .
\end{aligned}
$$$$
\text { soft-soft: }
$$

The four mass distributions are compared with each other in Fig. 2(a). Evidently, the gluino polarization leads to distinct patterns in the invariant jet-jet distributions.

\footnotetext{
${ }^{3}$ The far jets will be taken into account properly in the detailed phenomenology subsections.
} 
If no charges are measured, the minimal supersymmetric standard model Majorana theory predicts the moderately soft mass distribution, Eq. (1.5), of the near-quark jets in the gluino decays. In a gluino Dirac theory, on the other hand, pairs $\tilde{g}_{D} \tilde{g}_{D}^{c}$ are generated decaying to $\tilde{q}_{L} \tilde{q}_{L}^{*}$ but not $\tilde{q}_{L} \tilde{q}_{L}$ final states [8]. Such a theory would therefore predict an invariant mass distribution of the hard-hard and soft-soft types for aligned gluino spins. Thus, the shape of the mass distributions discriminates between standard Majorana and other Dirac supersymmetric theories.

2) Super-Compton process: Since the processes, Eq. (1.3), are maximally $P$ violating if $L$ and $R$ squarks are discriminated by their decay patterns, the produced gluinos are polarized. The polarization can be measured by exploiting the same methods for the first two and the third generation as outlined above. The polarization reflects itself in the invariant mass distribution of the near-quark jet in the gluino cascade with the primary squark decay jet, for asymptotic energies:

$$
\begin{aligned}
\text { hard: } \sigma^{-1} d \sigma / d m^{2} & =2\left(1-m^{2}\right) \\
\text { soft: } & =2\left[\log m^{-2}-\left(1-m^{2}\right)\right] .
\end{aligned}
$$

The two distributions are displayed in Fig. 2(b), exhibiting quite different shapes for the hard and soft decay configurations.

Switching from Majorana to Dirac gluinos does not affect the degree of gluino polarization. Nevertheless, as a result of $D$ conservation $\tilde{g}_{D}$ states are generated only in association with $\tilde{q}_{L}$ states, and $\tilde{g}_{D}^{c}$ states only in association with $\tilde{q}_{R}$ states, and $c c$ pairs correspondingly [8]. The decays of the $\tilde{g}_{D}^{(c)}$ particles follow the pattern discussed earlier.

The report includes two central sections. In Sec. II we analyze spin-spin correlations in gluino-pair production, followed by the discussion of the super-Compton process in Sec. III. In both sections we present analytical results at the parton level, and we illustrate the spin effects by calculating jet-jet invariant mass distributions for gluino production and decay at the LHC. While only the basic theoretical points are elaborated to emphasize the salient features of the spin correlations, the analysis nevertheless demonstrates the potential impact of spin correlations for high-precision supersymmetry studies at the LHC in the future. Section IV concludes the report.

\section{GLUINO POLARIZATION IN PAIR PRODUCTION}

\section{A. Gluino production at the parton level}

The $s$ - and $t$-channel exchange mechanisms, complemented by the $u$-channel exchanges, are shown in Figs. 1(a) and 1(b) for quark-antiquark and gluon-gluon collisions. For unpolarized beams the parton-parton cross sections are given by $[4,5]$

$$
\begin{aligned}
\sigma[q \bar{q} \rightarrow \tilde{g} \tilde{g}]= & \frac{\pi \alpha_{s}^{2}}{s} \beta\left(\frac{8}{9}+\frac{4 m_{\tilde{g}}^{2}}{9}\right)-\frac{\pi \alpha_{s} \hat{\alpha}_{s}}{s}\left[\left(\frac{2 m_{\tilde{g}}^{2}}{3}+\frac{m_{-}^{4}}{6}\right) L+\beta\left(\frac{4}{3}+\frac{2 m_{-}^{2}}{3}\right)\right]+\frac{\pi \hat{\alpha}_{s}^{2}}{s}\left[\left(\frac{16 m_{-}^{2}}{27}+\frac{4 m_{\tilde{g}}^{2}}{27\left(2-m_{-}^{2}\right)}\right) L\right. \\
& \left.+\beta\left(\frac{32}{27}+\frac{32 m_{-}^{4}}{27\left(4 m_{\tilde{q}}^{2}+m_{-}^{4}\right)}\right)\right], \\
\sigma[g g \rightarrow \tilde{g} \tilde{g}]= & \frac{\pi \alpha_{s}^{2}}{s}\left[\left(\frac{9}{4}+\frac{9 m_{\tilde{g}}^{2}}{4}-\frac{9 m_{\tilde{g}}^{4}}{16}\right) \log \left(\frac{1+\beta}{1-\beta}\right)-\beta\left(3+\frac{51 m_{\tilde{g}}^{2}}{16}\right)\right],
\end{aligned}
$$

where

$$
L=\log \frac{1+\beta-m_{-}^{2} / 2}{1-\beta-m_{-}^{2} / 2} \quad \text { with } \quad \beta=\sqrt{1-m_{\tilde{g}}^{2}}, \quad \alpha_{s}=g_{s}^{2} / 4 \pi \quad \text { and } \quad \hat{\alpha}_{s}=\hat{g}_{s}^{2} / 4 \pi,
$$

if the polarization of the gluinos in the final state is not measured. All masses are scaled by the parton beam energy $\sqrt{s} / 2$ in the parton-parton center of mass (c.m.). frame, i.e.

$$
m_{\tilde{g}, \tilde{q}}^{2}=4 M_{\tilde{g}, \tilde{q}}^{2} / s \quad \text { and } \quad m_{-}^{2}=4\left(M_{\tilde{g}}^{2}-M_{\tilde{q}}^{2}\right) / s .
$$

The couplings $g_{s}$ and $\hat{g}_{s}$ are the QCD gauge and Yukawa couplings, respectively, which are identical in super-QCD. Though noted here in Born approximation [4], the cross sections are known more accurately at next-to-leading order in super-QCD [5] and including threshold resummations [14]. The angular distributions for gluino-pair production read

$$
\begin{aligned}
& \frac{d \sigma}{d \Omega}[q \bar{q} \rightarrow \tilde{g} \tilde{g}]=\frac{\beta}{s}\left[\frac{\alpha_{s}^{2}}{6}\left(2+m_{\tilde{g}}^{2}-\kappa_{+} \kappa_{-}\right)+\frac{2 \alpha_{s} \hat{\alpha}_{s}}{3} \frac{m_{\tilde{g}}^{2}+\kappa_{+}^{2}}{m_{-}^{2}-2 \kappa_{+}}+\frac{\hat{\alpha}_{s}^{2}}{27}\left(\frac{32 \kappa_{+}^{2}}{\left(m_{-}^{2}-2 \kappa_{+}\right)^{2}}+\frac{4 m_{\tilde{g}}^{2}}{\left(m_{-}^{2}-2 \kappa_{+}\right)\left(m_{-}^{2}-2 \kappa_{-}\right)}\right)\right]^{\mathcal{S}}, \\
& \frac{d \sigma}{d \Omega}[g g \rightarrow \tilde{g} \tilde{g}]=\frac{\alpha_{s}^{2} \beta}{s} \frac{9}{32}\left[\frac{\kappa_{+}^{2} \kappa_{-}^{2}-2 m_{\tilde{g}}^{2}\left(m_{\tilde{g}}^{2}-1\right)}{2 \kappa_{+} \kappa_{-}}+2 \frac{\kappa_{+} \kappa_{-}-m_{\tilde{g}}^{2}\left(m_{\tilde{g}}^{2}-\kappa_{+}\right)}{\kappa_{+}^{2}}-\frac{2 \kappa_{+} \kappa_{-}+\left(\kappa_{+}-\kappa_{-}\right) m_{\tilde{g}}^{2}}{2 \kappa_{+}}\right]^{\mathcal{S}} .
\end{aligned}
$$


We use the definitions

$$
\kappa_{ \pm}=1 \pm \beta \cos \theta, \quad[F]^{\mathcal{S} / \mathcal{A}}=F(\cos \theta) \pm F(-\cos \theta),
$$

from this point on. The polar angle $\theta$ denotes the gluino flight direction with respect to the incoming particle momenta in the parton-parton c.m. frame. The angular distribution is forward-backward symmetric for the production of Majorana pairs. For the sake of brevity, the cross sections Eq. (2.2) will be denoted in the following by $\mathcal{N}_{q \bar{q}}$ and $\mathcal{N}_{g g}$ for the $q \bar{q}$ and $g g$ channels, respectively.

Quite generally, nontrivial polarization effects are generated in super-QCD introduced by the parity-violating quark-squark-gluino Yukawa couplings, while spin-spin correlations among the final-state gluinos are generated also by parity conserving gluon-gluino-gluino interactions. However, parity-violating effects in the first two generations of super-QCD are strongly suppressed by the small differences between the squark masses. In fact, the superQCD action is invariant under the $P$ transformation supplemented by $L / R$-squark exchange in the limit of equalmass $L / R$ squarks. Since the modulus of the potential gluino polarization vector $\left|M_{L}^{2}-M_{R}^{2}\right| /\left[M_{L}^{2}+M_{R}^{2}\right] \sim$ $10^{-2}$ is expected to be small [12], we neglect these effects in the present analysis.

Spin-spin correlations are conveniently described by the tensor $\mathcal{C}_{\mu \nu}$ following the formalism developed in Ref. [15]:

$$
\frac{d \sigma\left(s_{1}, s_{2}\right)}{d \Omega}=\frac{d \sigma}{d \Omega} \frac{1}{4}\left[1+\mathcal{C}_{\mu \nu} s_{1}^{\mu} s_{2}^{\nu}\right] .
$$

The two gluinos in the parton-parton c.m. frame are assigned the spin vectors $s_{1}$ and $s_{2}$; they are related to the spin vectors $\breve{s}_{1,2}$ in the gluino rest frames by respective Lorentz transformations $s_{1,2}=\Lambda_{1,2} \check{s}_{1,2}$, the corresponding matrix $\breve{\mathcal{C}}$ associated with the general spin-density matrix, see e.g. [16]. Choosing the $\hat{z}$ axis along one of the gluino flight directions, the $\hat{x}$ axis transverse to this vector within the production plane and pointing into the obtuse wedge between initial and final-state momenta, and the $\hat{y}$ axis normal to the production plane, the longitudinal, transverse, and normal spin vectors can be written as

$$
\begin{aligned}
& s_{1,2}^{l}=[\beta, 0,0, \pm 1] / m_{\tilde{g}}, \\
& s_{1,2}^{t}=[0, \pm 1,0,0], \quad \text { and } s_{1,2}^{n}=[0,0, \pm 1,0] .
\end{aligned}
$$

The longitudinal \pm components describe helicity + states of the two gluinos; the spin vectors of the helicity - states are given by $-s_{1,2}^{l}$. The matrix $\mathcal{C}$ is effectively $2 \times 2 \oplus 1 \times$ 1 dimensional; it is symmetric for Majorana gluinos and consists of four nontrivial components: $l l, l t=t l, t t ; n n$. Based on the correlation tensor, before orthogonalization with respect to the gluino momenta,

$$
\begin{aligned}
q \bar{q}: \mathcal{C}_{\mu \nu}= & \frac{\beta}{s}\left[\frac{\alpha_{s}^{2}}{6}\left(\beta^{2} \sin ^{2} \theta g_{\mu \nu}-2 \kappa_{+} k_{1, \mu} k_{2 \nu}\right)+\frac{\alpha_{s} \hat{\alpha}_{s}}{3} \frac{2 \beta^{2} \sin ^{2} \theta g_{\mu \nu}-\left(m_{\tilde{g}}^{2}+2 \kappa_{-}\right) k_{2 \mu} k_{1 \nu}+\left(m_{\tilde{g}}^{2}-2 \kappa_{+}\right) k_{1 \mu} k_{2 \nu}}{m_{-}^{2}-2 \kappa_{+}}\right. \\
& \left.-\frac{\hat{\alpha}_{s}^{2}}{27}\left(\frac{32 m_{\tilde{g}}^{2} k_{2 \mu} k_{1 \nu}}{\left(m_{-}^{2}-2 \kappa_{+}\right)^{2}}-\frac{4\left[\beta^{2} \sin ^{2} \theta g_{\mu \nu}+\left(m_{\tilde{g}}^{2}-2 \kappa_{+}\right) k_{1 \mu} k_{2 \nu}\right]}{\left(m_{-}^{2}-2 \kappa_{-}\right)\left(m_{-}^{2}-2 \kappa_{+}\right)}\right)\right]^{\mathcal{S}} / \mathcal{N}_{q \bar{q}}, \\
g g: \mathcal{C}_{\mu \nu}= & \frac{\alpha_{s}^{2} \beta}{s} \frac{9}{32}\left[\frac{1}{2}\left(-\kappa_{+} \kappa_{-} g_{\mu \nu}+2 \kappa_{+} k_{1 \mu} k_{2 \nu}\right)+\frac{2 m_{\tilde{g}}^{2}}{\kappa_{+}^{2}}\left(\left(m_{\tilde{g}}^{2}-\kappa_{+}\right) g_{\mu \nu}+k_{2 \mu} k_{1 \nu}\right)+\frac{1}{\kappa_{+} \kappa_{-}}\left(\left(m_{\tilde{g}}^{4}+\beta^{2} \sin ^{2} \theta\right) g_{\mu \nu}\right.\right. \\
& \left.\left.+\left(m_{\tilde{g}}^{2}-2 \kappa_{+}\right) k_{1 \mu} k_{2 \nu}\right)+\frac{1}{2 \kappa_{+}}\left(2\left(\kappa_{+} \kappa_{-}+m_{\tilde{g}}^{2} \beta \cos \theta\right) g_{\mu \nu}+\left(m_{\tilde{g}}^{2}-2 \kappa_{+}\right) k_{1 \mu} k_{2 \nu}-\left(m_{\tilde{g}}^{2}+2 \kappa_{-}\right) k_{2 \mu} k_{1 \nu}\right)\right]^{\mathcal{S}} / \mathcal{N}_{g g},
\end{aligned}
$$

with $[F]_{\mu \nu}^{\mathcal{S}}$ denoting the symmetrized tensor

$$
[F]_{\mu \nu}^{\mathcal{S}}=F_{\mu \nu}\left(\cos \theta, k_{1}, k_{2}\right)+F_{\mu \nu}\left(-\cos \theta, k_{2}, k_{1}\right)
$$

and $k_{1}, k_{2}$ being the initial parton 4-momenta, the spin matrix-elements can easily be derived: 


$$
\begin{aligned}
& q \bar{q} \text { channel: } \mathcal{C}^{l l}=-\frac{\beta}{s}\left[\frac{\alpha_{s}^{2}}{6}\left(\beta^{2}+\left(1+m_{\tilde{g}}^{2}\right) \cos ^{2} \theta\right)+\frac{2 \alpha_{s} \hat{\alpha}_{s}}{3} \frac{\beta^{2}+2 \beta \cos \theta+\left(1+m_{\tilde{g}}^{2}\right) \cos ^{2} \theta}{m_{-}^{2}-2 \kappa_{+}}\right. \\
& \left.+\hat{\alpha}_{s}^{2}\left(\frac{32}{27} \frac{(\beta+\cos \theta)^{2}}{\left(m_{-}^{2}-2 \kappa_{+}\right)^{2}}+\frac{4}{27} \frac{m_{\tilde{g}}^{2} \cos ^{2} \theta}{\left(m_{-}^{2}-2 \kappa_{-}\right)\left(m_{-}^{2}-2 \kappa_{+}\right)}\right)\right]^{\mathcal{S}} / \mathcal{N}_{q \bar{q}} \\
& \mathcal{C}^{l t}=\frac{\beta m_{\tilde{g}}}{s} \sin \theta\left[\frac{\alpha_{s}^{2}}{3} \cos \theta+\frac{2 \alpha_{s} \hat{\alpha}_{s}}{3} \frac{\beta+2 \cos \theta}{m_{-}^{2}-2 \kappa_{+}}\right. \\
& \left.+\hat{\alpha}_{s}^{2}\left(\frac{32}{27} \frac{\beta+\cos \theta}{\left(m_{-}^{2}-2 \kappa_{+}\right)^{2}}+\frac{4}{27} \frac{\cos \theta}{\left(m_{-}^{2}-2 \kappa_{+}\right)\left(m_{-}^{2}-2 \kappa_{-}\right)}\right)\right]^{\mathcal{A}} / \mathcal{N}_{q \bar{q}}, \\
& \mathcal{C}^{t t}=-\frac{\beta}{s} \sin ^{2} \theta\left[\frac{\alpha_{s}^{2}}{6}\left(m_{\tilde{g}}^{2}+1\right)+\frac{2 \alpha_{s} \hat{\alpha}_{s}}{3} \frac{m_{\tilde{g}}^{2}+1}{m_{-}^{2}-2 \kappa_{+}}\right. \\
& \left.+\hat{\alpha}_{s}^{2}\left(\frac{32}{27} \frac{m_{\tilde{\delta}}^{2}}{\left(m_{-}^{2}-2 \kappa_{+}\right)^{2}}+\frac{4}{27} \frac{1}{\left(m_{-}^{2}-2 \kappa_{-}\right)\left(m_{-}^{2}-2 \kappa_{+}\right)}\right)\right]^{\mathcal{S}} / \mathcal{N}_{q \bar{q}} \\
& \mathcal{C}^{n n}=\frac{\beta^{3}}{s} \sin ^{2} \theta\left[\frac{\alpha_{s}^{2}}{6}+\frac{2 \alpha_{s} \hat{\alpha}_{s}}{3} \frac{1}{m_{-}^{2}-2 \kappa_{+}}+\frac{4 \hat{\alpha}_{s}^{2}}{27} \frac{1}{\left(m_{-}^{2}-2 \kappa_{+}\right)\left(m_{-}^{2}-2 \kappa_{-}\right)}\right]^{\mathcal{S}} / \mathcal{N}_{q \bar{q}} \text {, }
\end{aligned}
$$

$g g$ channel: $\mathcal{C}^{l l}=\frac{9 \alpha_{s}^{2} \beta}{32 m_{\tilde{g}}^{2} s}\left[\kappa_{+}(\beta-\cos \theta)^{2}-\frac{\kappa_{+} \kappa_{-}}{2}\left(1+\beta^{2}\right)+\frac{2 m_{\tilde{g}}^{2}}{\kappa_{+}^{2}}\left((\beta+\cos \theta)^{2}+\left(1+\beta^{2}\right)\left(m_{\tilde{g}}^{2}-\kappa_{+}\right)\right)\right.$

$$
\begin{aligned}
& -\frac{1}{\kappa_{+} \kappa_{-}}\left(\left(2 \kappa_{-}-m_{\tilde{g}}^{2}\right)(\beta+\cos \theta)^{2}-\left(1+\beta^{2}\right)\left(m_{\tilde{g}}^{4}+\beta^{2} \sin ^{2} \theta\right)\right)+\frac{1}{2 \kappa_{+}}\left(\left(m_{\tilde{g}}^{2}-2 \kappa_{+}\right)(\beta-\cos \theta)^{2}\right. \\
& \left.\left.-\left(m_{\tilde{g}}^{2}+2 \kappa_{-}\right)(\beta+\cos \theta)^{2}+2\left(1+\beta^{2}\right)\left(\beta m_{\tilde{g}}^{2} \cos \theta+\kappa_{+} \kappa_{-}\right)\right)\right]^{\mathcal{S}} / \mathcal{N}_{g g}, \\
\mathcal{C}^{l t}= & -\frac{9 \alpha_{s}^{2} \beta}{32 s} \frac{\sin \theta}{m_{\tilde{g}}}\left[\kappa_{-}(\beta+\cos \theta)+\frac{2 m_{\tilde{g}}^{2}}{\kappa_{+}^{2}}(\beta+\cos \theta)-\frac{1}{\kappa_{+} \kappa_{-}}(\beta+\cos \theta)\left(2 \kappa_{-}-m_{\tilde{g}}^{2}\right)\right. \\
& \left.-\frac{m_{\tilde{g}}^{2}}{\kappa_{+}}(\beta+2 \cos \theta)\right]^{\mathcal{A}} / \mathcal{N}_{g g}, \\
\mathcal{C}^{t t}= & \frac{9 \alpha_{s}^{2} \beta}{32 s}\left[\kappa_{+}\left(\sin ^{2} \theta-\frac{\kappa_{-}}{2}\right)+\frac{2 m_{\tilde{g}}^{2}}{\kappa_{+}^{2}}\left(m_{\tilde{g}}^{2}+\sin ^{2} \theta-\kappa_{+}\right)-\frac{1}{\kappa_{+} \kappa_{-}}\left(\left(2 \kappa_{+}-1\right) \sin ^{2} \theta-m_{\tilde{g}}^{4}\right)\right. \\
& \left.+\frac{1}{\kappa_{+}}\left(\beta m_{\tilde{g}}^{2} \cos \theta+\kappa_{+} \kappa_{-}-2 \sin ^{2} \theta\right)\right]^{\mathcal{S}} / \mathcal{N}_{g g}, \\
\mathcal{C}^{n n}= & -\frac{9 \alpha_{s}^{2} \beta}{32 s}\left[\frac{\kappa_{+} \kappa_{-}}{2}-\frac{2 m_{\tilde{g}}^{2}\left(m_{\tilde{g}}^{2}-\kappa_{+}\right)}{\kappa_{+}^{2}}-\frac{m_{\tilde{g}}^{4}+\beta^{2} \sin ^{2} \theta}{\kappa_{+} \kappa_{-}}-\frac{\kappa_{+} \kappa_{-}+m_{\tilde{g}}^{2} \beta \cos \theta}{\kappa_{+}}\right]^{\mathcal{S}} / \mathcal{N}_{g g} .
\end{aligned}
$$

The index $l$ denotes positive gluino helicity. The four correlation matrix-elements are displayed for $\sqrt{s}=$ $2 \mathrm{TeV}$ and SPS1a $\mathrm{a}^{\prime}$ masses $M_{\tilde{g}}=607 \mathrm{GeV}$ and $M_{\tilde{q}}=$ $M_{\tilde{u}_{L}}=565 \mathrm{GeV}$ [7] in Figs. 3(a) and 3(b) in the $q \bar{q}$ and $g g$ channels, respectively. The antisymmetric terms drop out if $q \bar{q} \oplus \bar{q} q$ channels are summed up for the symmetric $p p$ kinematics. As expected, $\mathcal{C}^{l l}$, corresponding to $S_{z}=0$ for two equal gluino helicities in $q \bar{q} \rightarrow \tilde{g} \tilde{g}$, approaches -1 for forward/backward production, lifted (in particular by means of the $t / u$ exchange $\tilde{u}, \tilde{d}$ propagators) to larger values in between for nonzero gluino masses. $\mathcal{C}^{t t}$, on the other hand, is maximal for perpendicular production. The correlation of the normal polarizations is negative for nonzero production angles but, as in the transverse direction, approaches zero for forward production. The $S_{z}=0$ com- ponent of the gluon-gluon spin wave function in $g g \rightarrow \tilde{g} \tilde{g}$ gives rise to maximal forward production of $S_{z}=0$ gluino pairs and, as expected, $\mathcal{C}^{t t}$ and $\mathcal{C}^{n n}$ approach equal values for forward production.

\section{B. Gluino decays}

If gluinos are heavier than squarks, they decay to quarks and squarks [17]. The squarks decay subsequently to quarks plus neutralinos or charginos. Particularly at the reference point SPS1a/a' the lightest neutralino $\tilde{\chi}_{1}^{0}$ is almost a pure bino state, while $\tilde{\chi}_{2}^{0}$ is almost a pure wino state, like the chargino $\tilde{\chi}_{1}^{ \pm}$. The heavier neutralinos and charginos are Higgsino-like states, and they do not play a significant role for matter particles of the first two generations. $R$ squarks will therefore decay predominantly 


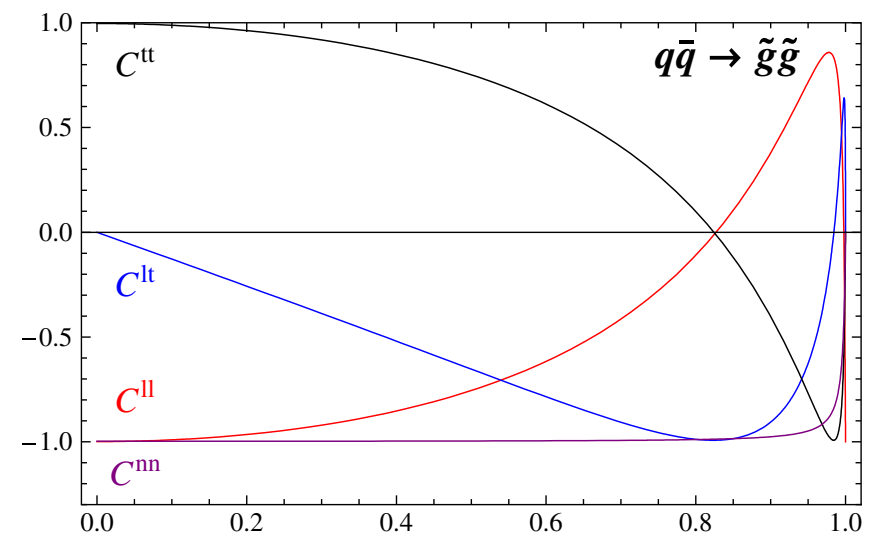

(a)

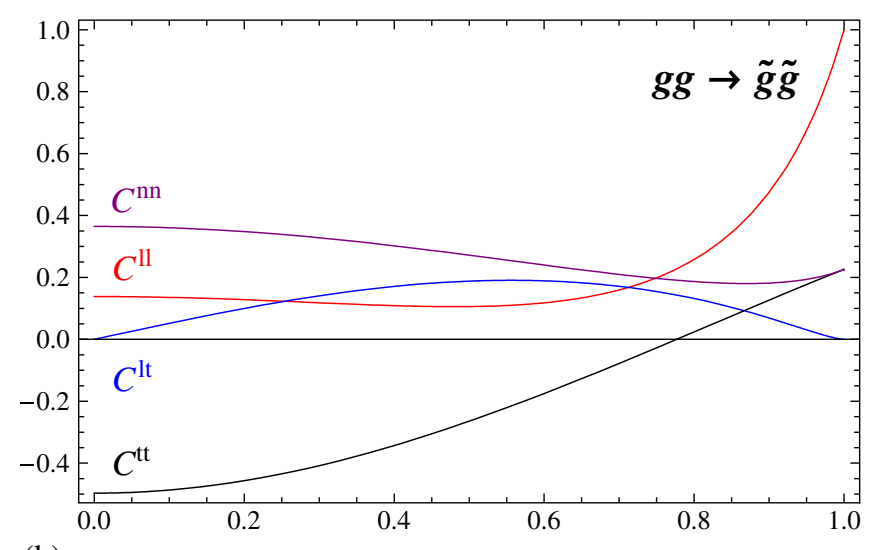

(b)

$\cos (\Theta)$

FIG. 3 (color online). Correlation matrix elements for $\sqrt{s}=$ $2 \mathrm{TeV}$ in the quark annihilation (a) and gluon fusion (b) channels.

into the lightest neutralino $\tilde{\chi}_{1}^{0}$ as the lightest supersymmetric particle, while $L$ sqarks decay preferentially to the heavier neutralino $\tilde{\chi}_{2}^{0}$ or chargino $\tilde{\chi}_{1}^{ \pm}$, followed by subsequent $\tilde{\chi}$ cascades. The decay branching ratios [18] are collected in the following set for the reference point SPS1a':

$$
\begin{array}{rlrl}
\Gamma\left[\tilde{g} \rightarrow q \tilde{q}_{L}\right] & =7.73 \%, & \Gamma\left[\tilde{g} \rightarrow q \tilde{q}_{R}\right]=17.0 \%, & q \neq b, t, \\
\Gamma\left[\tilde{g} \rightarrow b \tilde{b}_{1}\right] & =10.8 \%, & \Gamma\left[\tilde{g} \rightarrow b \tilde{b}_{2}\right]=4.67 \%, \\
\Gamma\left[\tilde{g} \rightarrow t \tilde{t}_{1}\right] & =9.81 \% .
\end{array}
$$

The decay $\tilde{g} \rightarrow t \tilde{t}_{2}$ is kinematically forbidden.

If the gluinos are polarized along the axis $\overrightarrow{\breve{s}}$ in the rest frame, final-state distributions are determined by the polarization vector $\mathcal{P}_{\mu}$,

$$
d \Gamma=d \Gamma^{\mathrm{unpol}}\left[1-\mathcal{P}_{\mu} s_{\mu}\right],
$$

in a general Lorentz frame $\check{s}_{\mu} \rightarrow s_{\mu}$.

For gluinos polarized with degree unity, the angular distribution of the quark jets with respect to the spin axis depends on the particle/antiparticle character of the squarks, for the first two generations:

$$
\begin{gathered}
\frac{1}{\Gamma} \frac{d \Gamma}{d \cos \theta_{*}}\left[\tilde{g} \rightarrow q_{R, L} \tilde{q}_{R, L}^{*}\right]=\frac{1}{2}\left[1 \pm \cos \theta_{*}\right], \\
\frac{1}{\Gamma} \frac{d \Gamma}{d \cos \theta_{*}}\left[\tilde{g} \rightarrow \bar{q}_{L, R} \tilde{q}_{R, L}\right]=\frac{1}{2}\left[1 \mp \cos \theta_{*}\right] .
\end{gathered}
$$

The mass eigenstates of sparticles of the third generation, stop particles, in particular, are mixtures of $R, L$ current eigenstates [19]. Denoting the $\tilde{t}_{R}$ component of the (light) $\tilde{t}_{1}$ wave function by $\cos \theta_{\tilde{t}}$, the coefficients of the $\cos \theta$ terms in Eqs. (2.12) are altered from unity to

$$
\begin{aligned}
& \alpha_{\tilde{t}_{1,2}}= \pm \cos 2 \theta_{\tilde{t}} \beta_{t} \kappa_{\tilde{t}_{1,2}}^{-1}, \\
& \kappa_{\tilde{t}_{1,2}}=1 \pm 2 \sin 2 \theta_{\tilde{t}}\left(1-\beta_{t}^{2}\right)^{1 / 2},
\end{aligned}
$$

where $\beta_{t}$ is the velocity of the top quark in the gluino rest frame. As expected, nonzero top mass and mixing dilute the polarization effects.

As discussed previously, the spin information is washed out in inclusive analyses of the final states. Adding up quarks/antisquarks and antiquarks/squarks, the $\cos \theta$-dependent terms cancel each other. These final states can be discriminated however by tagging either the charge of the quark/antiquark $t, b$ in the third generation, or by tagging the squark/antisquark by measuring the charges in chargino decays. This method can successfully be applied within the first (second) generation only if up- and downstates can be distinguished since the states $\tilde{u}_{L}$ and $\tilde{d}_{L}^{*}$ generate final states with the same charge topology but opposite quark/antiquark helicities. If, however, their decay branching ratios are different, the spin-dependent contributions of the two channels do not add up to zero anymore but come with the spin-analysis power $\kappa=$ $\left|B R_{\tilde{d}}-B R_{\tilde{u}}\right| /\left[B R_{\tilde{d}}+B R_{\tilde{d}}\right] \neq 0$. Though the partial widths for decays to charginos are the same by isospin invariance, the total widths of $\tilde{u}$ and $\tilde{d} / \tilde{d}^{*}$ may be different, nevertheless, for $\tilde{\chi}_{2}^{0}$ and $\tilde{\chi}_{1}^{0}$ not being pure wino and bino states [20]. The spin-analysis power $\kappa$, which can vary in principle between +1 and -1 , reaches a value of $1 / 5$, for instance, if isovector and isoscalar components of the neutralinos are mixed maximally and decays to $\tilde{\chi}_{2}^{0}$ are phase-space suppressed to $1 / 2$ with regard to $\tilde{\chi}_{1}^{0}$.

\section{Bypass: The dirac alternative}

If the gluinos were Dirac particles [8] as formulated in $N=2$ hybrid models, they could only be produced in the fermion-antifermion mode of gluino-pair production:

$$
q \bar{q}, \quad g g \rightarrow \tilde{g}_{D} \tilde{g}_{D}^{c} .
$$

The decay channels would be restricted, by conservation of the Dirac charge, to

$$
\begin{aligned}
& \tilde{g}_{D} \rightarrow \bar{q}_{L} \tilde{q}_{R} \text { and } q_{L} \tilde{q}_{L}^{*}, \\
& \tilde{g}_{D}^{c} \rightarrow \bar{q}_{R} \tilde{q}_{L} \text { and } q_{R} \tilde{q}_{R}^{*} .
\end{aligned}
$$


Assuming, as done commonly, the $\tilde{q}_{R}, \tilde{q}_{R}^{*}$ squarks to decay into the lightest, invisible neutralino $\tilde{\chi}_{1}^{0}=\mathrm{LSP}$, and tagging the $\tilde{q}_{L}, \tilde{q}_{L}^{*}$ squarks, the spins of the individual gluinos $\tilde{g}$ and $\tilde{g}^{c}$ can be reconstructed with spin-analysis power 1 in the Dirac theory. Unlike the Majorana theory, quark and antiquark always come with opposite helicities in pairs.

\section{Spin-phenomenology of gluino-pair production}

The spin-correlation effects in gluino-pair production will be illustrated by the analysis of jet-jet invariant masses as a simple indicator. Since spin correlations will play only a role in precision measurements at LHC, our illustration is designed to be of qualitative theoretical nature, without cuts on observables and QCD radiative corrections, etc. Thus, only a coarse picture of spin effects will be presented for illustration as (semi)realistic experimental simulations are far beyond the scope of this theoretical study.

Quite generally, spin correlation effects are described in the process $p p \rightarrow \tilde{g} \tilde{g} \rightarrow$ final state by the production correlation matrix $\mathcal{C}$ and the two decay polarization vectors $\mathcal{P}_{1}$ and $\mathcal{P}_{2}[15]$ :

$$
d \sigma=d \sigma^{\mathrm{unpol}}\left[1+\mathcal{C}_{\mu \nu} \mathcal{P}_{1 \mu} \mathcal{P}_{2 \nu}\right]
$$

The spin correlation affects, in principle, all final-state observables.

The decay chains of the two gluinos, cf. Fig. 4,

$$
\tilde{g}_{1} \tilde{g}_{2} \rightarrow\left[\bar{q}_{1} \tilde{q}_{1}\right]\left[\bar{q}_{2} \tilde{q}_{2}\right] \rightarrow \bar{q}_{1} q_{1} \bar{q}_{2} q_{2} \tilde{\chi} \tilde{\chi},
$$

give rise to six invariant masses $M_{i j}^{2}=\left(p_{i}+p_{j}\right)^{2}$ which can be formed within the four-jet ensemble of the final state.

The invariant mass distribution of the two near jets, labeled generically by $\bar{q}_{1}$ and $\bar{q}_{2}$, is most sensitive to the gluino polarization. While it will not be known in practice which of the observed final-state jets are associated with the near jets in the gluino decays, in the vast majority of events for SPS1a/a'-type mass configurations these two jets can be identified with the jets of minimal transverse momentum. Ordering, therefore, the jets $j_{1}$ to $j_{4}$ according to rising transverse momenta, the $j_{1} j_{2}$ combination is expected to retain most of the sensitivity to spin correlations. (Specific kinematical properties of events must be exploited in SPS1a/a'-type and other scenarios to isolate

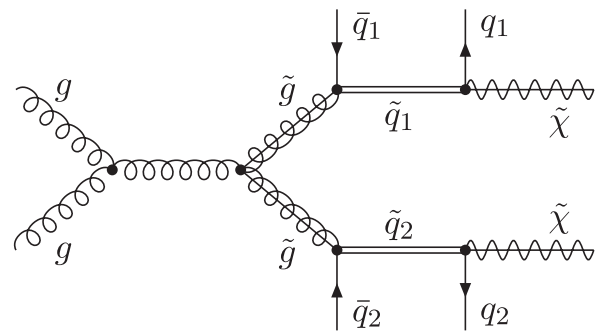

FIG. 4. Squark decay chain in gluino-pair production; chains involving antisquarks are to be added. spin effects, and the studies must be tailored carefully to the specific scenario and the corresponding kinematical configuration being analyzed.) Note that for gluino Majorana theories the $\bar{q}_{1} \bar{q}_{2}$ combination comes in all possible configurations: $\bar{q}_{R} \tilde{q}_{L}$ with $\bar{q}_{R} \tilde{q}_{L}$ and $q_{L} \tilde{q}_{L}^{*}$, i.e. equalhelicity as well as opposite-helicity (anti)quark states generating the low- $p_{\perp}$ jets. In contrast, Dirac theories would only allow opposite-helicity (anti)quark states generating these jets.

To illustrate the effect of spin correlations we compare the jet-jet invariant mass distributions for $\tilde{u}_{L} \tilde{u}_{L}$ and $\tilde{u}_{L} \tilde{u}_{L}^{*}$ intermediate states, associated with $\bar{q}_{R} \bar{q}_{R}$ and $\bar{q}_{R} q_{L}$ nearquark jets. The $L$ squarks $\tilde{u}_{L}, \tilde{u}_{L}^{*}$ can be tagged by observing leptonic decays of $\tilde{\chi}_{1}^{ \pm}$and $\tilde{\chi}_{2}^{0}$, which discriminate $L$ squarks from $R$ squarks decaying to the invisible $\tilde{\chi}_{1}^{0}$, cf. Ref. [8]. By tagging the $L$ squarks, kinematical effects due to different $L / R$ squark masses, with size similar to the spin effects, are eliminated. The spin-correlations will manifest themselves in different values of the jet-jet invariant masses, which depend on the relative orientation of the gluino spins. The average values $\left\langle M^{2}\right\rangle=\left(\left\langle M_{\tilde{u}_{L} \tilde{u}_{L}}^{2}\right\rangle+\right.$ $\left.\left\langle M_{\tilde{u}_{L} \tilde{u}_{L}^{*}}^{2}\right\rangle\right) / 2$ and the differences $\Delta M^{2}=\left|\left\langle M_{\tilde{u}_{L} \tilde{u}_{L}}^{2}\right\rangle-\left\langle M_{\tilde{u}_{L} \tilde{u}_{L}^{*}}^{2}\right\rangle\right|$ (the indices characterizing the intermediate squarks) are presented in Table I for all six jet-jet invariant masses. The numerical results have been obtained for the SPS1a' scenario with masses $M_{\tilde{g}}=607 \mathrm{GeV}, M_{\tilde{u}_{L}}=565 \mathrm{GeV}$, and $M_{\tilde{\chi}}=M_{\tilde{\chi}_{1}^{0}}=98 \mathrm{GeV}$. The CTEQ6L1 LO parton densities [21] have been adopted with the corresponding leadingorder $\alpha_{s}$, and all scales have been set to $\mu=M_{\tilde{g}}$. All numerical results presented in this section and below have been compared with results obtained using MADGRAPH/MADEVENT [22]; the results do agree with each other.

In the upper section of Table I invariant masses for identified jets are shown, and in the lower section they are shown for jets ordered according to transverse momenta. For the SPS1a/a'-type mass configurations considered here, the invariant mass distributions involving near jets from the $\tilde{g} \rightarrow q \tilde{q}$ decays are significantly softer than those involving far jets from $\tilde{q} \rightarrow q \tilde{\chi}$ decays. The gluino

TABLE I. Invariant jet-jet masses for gluino-pair production and decay. The average values $\left\langle M^{2}\right\rangle$ and the differences $\Delta M^{2}$ of the invariant mass distributions for $\tilde{u}_{L} \tilde{u}_{L}$ and $\tilde{u}_{L} \tilde{u}_{L}^{*}$ intermediate states are shown for identified jets (upper section) and for jets ordered according to transverse momenta (lower section).

\begin{tabular}{lccrrrr}
\hline \hline \multicolumn{2}{l}{ Gluino pairs: $\tilde{g} \tilde{g} \rightarrow[\bar{q} \tilde{q}][\bar{q} \tilde{q}] \rightarrow$} & $\bar{q}_{1} q_{1} \bar{q}_{2} q_{2} \tilde{\chi} \tilde{\chi}$ & & \\
Original quarks & $\bar{q}_{1} q_{1}$ & $\bar{q}_{1} \bar{q}_{2}$ & $\bar{q}_{1} q_{2}$ & $q_{1} \bar{q}_{2}$ & $q_{1} q_{2}$ & $\bar{q}_{2} q_{2}$ \\
\hline$\left\langle M^{2}\right\rangle\left[10^{3} \mathrm{GeV}^{2}\right]$ & 23.9 & 9.17 & 62.4 & 62.2 & 423 & 23.9 \\
$\Delta M^{2} /\left\langle M^{2}\right\rangle[\%]$ & $<0.1$ & 10.8 & 0.8 & 0.8 & $<0.1$ & $<0.1$ \\
$p_{\perp}$ ordered jets & $j_{1} j_{2}$ & $j_{1} j_{3}$ & $j_{1} j_{4}$ & $j_{2} j_{3}$ & $j_{2} j_{4}$ & $j_{3} j_{4}$ \\
$\left\langle M^{2}\right\rangle\left[10^{3} \mathrm{GeV}^{2}\right]$ & 9.56 & 30.1 & 43.7 & 39.6 & 64.7 & 417 \\
$\Delta M^{2} /\left\langle M^{2}\right\rangle[\%]$ & 10.2 & 2.4 & 1.7 & 2.0 & 3.0 & $<0.1$ \\
\hline \hline
\end{tabular}




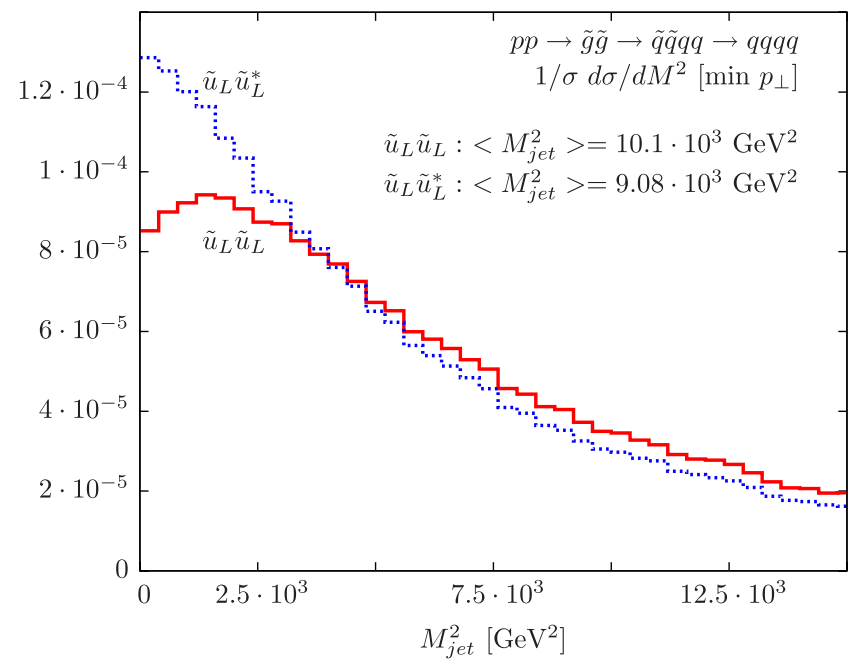

(a)

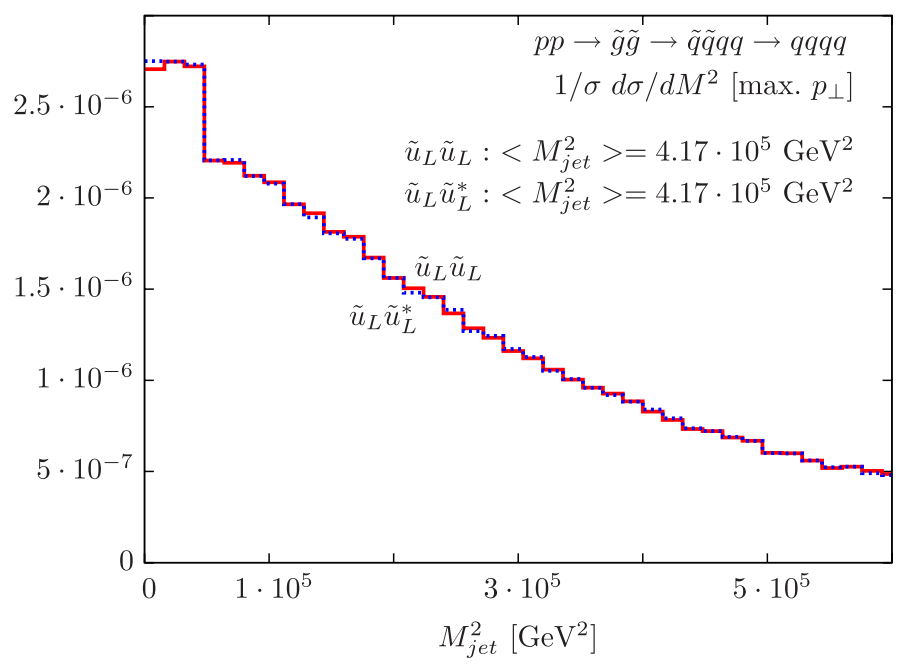

(b)

FIG. 5 (color online). Mass distribution of (a) the two jets with the lowest $p_{\perp}$, for which maximal spin correlations are predicted, and (b) the two jets with the highest $p_{\perp}$ where spin effects, within quark pairs generated almost exclusively by scalar decays, are expected to be very small.

polarization affects the invariant mass distribution involving the near jets, with a relative difference between $\tilde{u}_{L} \tilde{u}_{L}$ and $\tilde{u}_{L} \tilde{u}_{L}^{*}$ intermediate states of about $10 \%$. For all other invariant mass combinations the polarization effects are negligible. As evident from the lower section of the table, the average invariant mass and the invariant mass difference for the two jets with the smallest transverse momentum $j_{1} j_{2}$ are very close to the corresponding $\bar{q}_{1} \bar{q}_{2}$ jet values, in concordance with general expectations derived from the kinematics associated with $\tilde{q}, \tilde{g}$ mass parameters chosen in this example.

The differential distribution of the $p_{\perp}$-ordered jet-jet invariant mass $M_{j_{1} j_{2}}^{2}$ is depicted in Fig. 5(a) for constructive spin correlation and contrasted with destructive correlation. Correlations among jets generated almost exclusively in scalar squark decays are tiny as evident from the $M_{j_{3} j_{4}}^{2}$ distributions shown in Fig. 5(b). Without working out the details it should be noted that cuts on the minimal missing energy could be used to eliminate standard QCD stray jets. Lower $p_{\perp}$ cuts between 50 and $100 \mathrm{GeV}$ suppress additional QCD bremsstrahl jets emitted in the supersymmetric parton process itself. While such cuts are essential for reducing combinatorics, the impact on the signal cross section varies around 50\%. The impact on the spin correlations, measured by the ratio $\Delta M 2 /\langle M 2\rangle$ for the $j_{1} j_{2}$ invariant masses quoted in the table is changed however very little when such cuts are applied.

\section{SUPER-COMPTON PROCESS}

\section{A. Parton level}

Single polarization in gluino pair production has been proved in the foregoing section to be strongly suppressed at the squark-mass level $\sim\left|M_{L}^{2}-M_{R}^{2}\right| /\left[M_{L}^{2}+M_{R}^{2}\right]$ for the two light generations since the production process becomes effectively parity-even in the limit $M_{L} \rightarrow M_{R}$. However, if the squarks are produced as final particles, the $L / R$ character can be identified and the parity violation in the Yukawa vertex becomes effective. For example, $R$ squarks may decay into the invisible lightest supersymmetric particle while $L$ squarks can be marked by chargino decays. This constellation is realized in the super-Compton process Eq. (1.3).

Symmetrizing $q g \oplus g q$ as relevant for parton collisions at the symmetric $p p$ collider LHC, the spin-summed total cross section $[4,5,23]$ may be written

$$
\begin{aligned}
\sigma\left[q g \rightarrow \tilde{q}_{R, L} \tilde{g}\right]= & \frac{\pi \alpha_{s} \hat{\alpha}_{s}}{s}\left[\left(1+\frac{m_{-}^{2}}{2}-\frac{m_{\tilde{q}}^{2} m_{-}^{2}}{8}\right) L_{+}\right. \\
& +\left(\frac{2 m_{-}^{2}}{9}-\frac{m_{\tilde{q}}^{2} m_{-}^{2}}{8}-\frac{m_{-}^{4}}{18}\right) L_{-} \\
& \left.-p\left(\frac{7}{9}+\frac{32 m_{-}^{2}}{9}\right)\right],
\end{aligned}
$$

with

$$
L_{ \pm}=\log \frac{1+p \pm m_{-}^{2} / 4}{1-p \pm m_{-}^{2} / 4}
$$

while the angular dependence reads 


$$
\begin{aligned}
\frac{d \sigma}{d \Omega}\left[q g \rightarrow \tilde{q}_{R, L} \tilde{g}\right]= & \frac{\alpha_{s} \hat{\alpha}_{s} p}{s}\left[\frac{1}{36} \kappa_{\tilde{g}_{-}}-\frac{1}{36 \kappa_{\tilde{q}_{+}}^{2}}\left(m_{\tilde{q}}^{2}-\kappa_{\tilde{q}_{+}}\right)\left(m_{-}^{2}+2 \kappa_{\tilde{q}_{+}}\right)-\frac{1}{16 \kappa_{\tilde{g}_{-}}^{2}}\left[m_{\tilde{g}}^{2}\left(m_{-}^{2}-2 \kappa_{\tilde{g}_{-}}\right)-2 \kappa_{\tilde{g}_{-}}\left(\kappa_{\tilde{g}_{-}}+\kappa_{\tilde{q}_{+}}\right)\right]\right. \\
& +\frac{1}{576 \kappa_{\tilde{q}_{+}}}\left[m_{-}^{4}-2\left(m_{\tilde{g}}^{2}+m_{\tilde{q}}^{2}\right)+2 \kappa_{q_{+}}\left(m_{-}^{2}+2\right)\right]+\frac{1}{64 \kappa_{\tilde{g}_{-}}}\left[m_{\tilde{g}}^{4}-2 m_{\tilde{g}}^{2}\left(m_{\tilde{q}}^{2}+\kappa_{\tilde{g}_{-}}\right)\right. \\
& \left.\left.+\left(m_{\tilde{q}}^{2}-4\right)\left(m_{\tilde{q}}^{2}+2 \kappa_{\tilde{g}_{-}}\right)\right]+\frac{1}{32 \kappa_{\tilde{q}_{+}} \kappa_{\tilde{g}_{-}}}\left[m_{\tilde{q}}^{4}-m_{\tilde{g}}^{4}+\kappa_{\tilde{g}_{-}}\left(m_{\tilde{q}}^{2}+m_{\tilde{g}}^{2}\right)-2 \kappa_{\tilde{q}_{+}}\left(m_{\tilde{q}_{-}}^{2}+\kappa_{\tilde{g}_{-}}\right)\right]\right]^{\mathcal{S}}
\end{aligned}
$$

where the abbreviations

$$
\kappa_{\tilde{q}_{ \pm}}=\epsilon_{\tilde{q}}\left(1 \pm \beta_{\tilde{q}} \cos \theta\right),
$$

and

$$
\begin{gathered}
m_{\tilde{q}}=2 M_{\tilde{q}} / \sqrt{s}, \quad \epsilon_{\tilde{q}}=2 E_{\tilde{q}} / \sqrt{s}=1+\left(m_{\tilde{q}}^{2}-m_{\tilde{g}}^{2}\right) / 4, \\
\beta_{\tilde{q}}=p / \epsilon_{\tilde{q}}, \quad p=\sqrt{\left[1-\left(m_{\tilde{q}}+m_{\tilde{g}}\right)^{2} / 4\right]\left[1-\left(m_{\tilde{q}}-m_{\tilde{g}}\right)^{2} / 4\right]},
\end{gathered}
$$

have been used; $\tilde{q} \Leftrightarrow \tilde{g}$ correspondingly. These spinaveraged cross sections, discussed in detail in Ref. [5], are form-identical for $\tilde{q}_{L}$ and $\tilde{q}_{R}$ production.

The polarization vector of the gluino,

$$
\frac{d \sigma(s)}{d \Omega}=\frac{d \sigma}{d \Omega} \frac{1}{2}\left[1-\mathcal{C}_{\mu} s^{\mu}\right]
$$

using the spin-vector notation within the frame introduced before, can easily be determined:

$$
\begin{aligned}
\mathcal{C}_{\mu}= & \frac{\alpha_{s} \hat{\alpha}_{s} p m_{\tilde{g}}}{s}\left[-\frac{1}{36} k_{1 \mu}+\frac{1}{8 \kappa_{\tilde{g}_{-}}^{2}}\left(\kappa_{\tilde{g}_{+}}-2\right) k_{1 \mu}+\left(m_{\tilde{g}}^{2}-\kappa_{\tilde{g}_{-}}\right) k_{2 \mu}\right)+\frac{1}{72 \kappa_{\tilde{q}_{+}}^{2}}\left[m_{\tilde{g}}^{2}+m_{\tilde{q}}^{2}-2\left(p^{2}-\epsilon_{\tilde{q}}^{2}+2(1+p \cos \theta)\right)\right] k_{2 \mu} \\
& -\frac{1}{32 \kappa_{\tilde{g}_{-}}}\left[\left(\kappa_{\tilde{g}_{+}}-4\right) k_{1 \mu}+\left(\kappa_{\tilde{g}_{+}}-2 \epsilon_{\tilde{q}}\right) k_{2 \mu}\right]-\frac{1}{576 \kappa_{\tilde{q}_{+}}}\left[2\left(2+p \cos \theta-\epsilon_{\tilde{q}}\right) k_{1 \mu}+2\left(4-3 \epsilon_{\tilde{q}}+p \cos \theta\right) k_{2 \mu}\right] \\
& \left.+\frac{1}{32 \kappa_{\tilde{g}_{-}} \kappa_{\tilde{q}_{+}}}\left[2\left(2+p \cos \theta-\epsilon_{\tilde{q}}\right) k_{1 \mu}+2\left(m_{\tilde{g}}^{2}-p^{2}+\epsilon_{\tilde{q}}^{2}\right) k_{2 \mu}\right]\right]^{\mathcal{S}} / \mathcal{N}_{q g} .
\end{aligned}
$$

$\mathcal{N}_{q g}$ denotes the symmetrized angular distribution Eq. (3.3). Evaluation of the vector in the gluino rest frame yields the final results for the longitudinal, transverse, and normal components:

$$
\begin{aligned}
\mathcal{P}^{l}= & \frac{\alpha_{s} \hat{\alpha}_{s} p}{s}\left[\frac{1}{36}\left(\epsilon_{\tilde{g}} \cos \theta-p\right)+\frac{1}{8 \kappa_{\tilde{g}}^{2}}\left[\left(2 \epsilon_{\tilde{g}}-\epsilon_{\tilde{q}} m_{\tilde{g}}^{2}\right) \cos \theta+p\left(m_{\tilde{g}}^{2}-2\right)\right]-\frac{1}{72 \kappa_{\tilde{q}_{+}}^{2}}\left[( p + \epsilon _ { \tilde { g } } \operatorname { c o s } \theta ) \left(3\left(2 \epsilon_{\tilde{g}}-m_{\tilde{g}}^{2}\right)\right.\right.\right. \\
& \left.\left.-\left(2 \epsilon_{\tilde{q}}+m_{\tilde{q}}^{2}\right)+4 p \cos \theta\right)\right]-\frac{1}{16 \kappa_{\tilde{g}}}\left[\left(p^{2}+\epsilon_{\tilde{g}}^{2}\right) \cos \theta-2 p \epsilon_{\tilde{q}}\right]-\frac{1}{288 \kappa_{\tilde{q}_{+}}}\left[2\left(p^{2}+\epsilon_{\tilde{g}}\left(1-\epsilon_{\tilde{q}}\right)\right) \cos \theta\right. \\
& \left.\left.+2 p\left(3-2 \epsilon_{\tilde{q}}\right)\right]-\frac{1}{16 \kappa_{\tilde{q}_{+}} \kappa_{\tilde{g}}}\left[\epsilon_{\tilde{g}} p \cos ^{2} \theta-\frac{p}{4}\left(5 m_{\tilde{g}}^{2}+3 m_{\tilde{q}}^{2}+4\right)+\frac{1}{4}\left(m_{\tilde{q}}^{4}-m_{\tilde{g}}^{4}-4 m_{\tilde{q}}^{2}\right) \cos \theta\right]\right]^{\mathcal{S}} / \mathcal{N}_{q g}, \\
\mathcal{P}^{t}= & \frac{\alpha_{s} \hat{\alpha}_{s} p m_{\tilde{g}} \sin \theta}{s}\left[\frac{1}{8 \kappa_{\tilde{g}_{-}}^{2}}\left[2\left(\epsilon_{\tilde{g}}-1\right)-m_{\tilde{g}}^{2}\right]-\frac{1}{72 \kappa_{\tilde{q}_{+}}^{2}}\left[3\left(m_{\tilde{g}}^{2}-2 \epsilon_{\tilde{g}}\right)+\left(m_{\tilde{q}}^{2}+2 \epsilon_{\tilde{q}}\right)-4 p \cos \theta\right]\right. \\
& \left.+\frac{\epsilon_{\tilde{g}}}{16 \kappa_{\tilde{g}_{-}}}+\frac{\epsilon_{\tilde{g}}-\epsilon_{\tilde{q}}}{288 \kappa_{\tilde{q}_{+}}}-\frac{1}{16 \kappa_{\tilde{q}_{+}} \kappa_{\tilde{g}_{-}}}\left[2\left(m_{\tilde{g}}^{2}-2 \epsilon_{\tilde{g}}\right)+\epsilon_{\tilde{q}}+2-p \cos \theta\right]\right]^{\mathcal{A}} / \mathcal{N}_{q g}, \\
\mathcal{P}^{n}= & 0,
\end{aligned}
$$

at the Born level for $R$ squarks in the final state, while the overall signs flip for $L$ squarks. The nontrivial longitudinal and transverse components of the polarization vector are displayed in Fig. 6 for the parton invariant energy $\sqrt{s}=$ $2 \mathrm{TeV}$ and the SPS1a' masses $M_{\tilde{g}}=607 \mathrm{GeV}$ and $M_{\tilde{q}}=$ $M_{\tilde{u}_{R}}=547 \mathrm{GeV}$. While the transverse polarization is very small for all production angles, the maximal parity violation of the Yukawa vertex renders the degree of longitudinal polarization large in the forward direction.
Normal gluino polarization is zero in Born approximation, but it can be generated by vertex loop-corrections which render the transition matrix formally $T$ odd. For example, the triangular $\tilde{q} \tilde{g} g$ super-QCD vertex correction to the amplitude $g q \rightarrow \tilde{g} \tilde{q}$ is complex, but the size of the normal polarization remains suppressed as a higher-order effect [15] at a level of $\alpha_{s} \tilde{M} / \sqrt{s}$, with $\tilde{M}$ denoting the typical supersymmetry mass scale. 


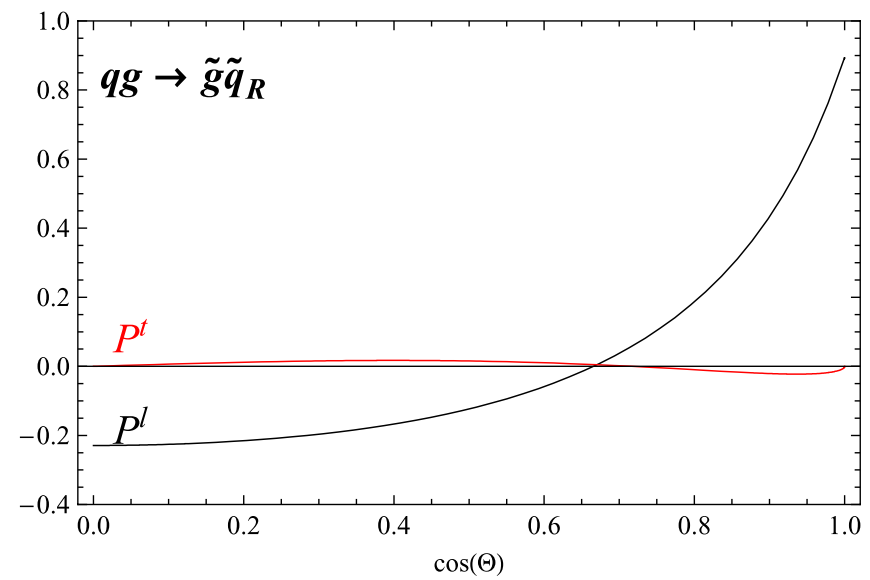

FIG. 6 (color online). Longitudinal and transverse components of the gluino polarization vector for $R$ squarks in the final state of the super-Compton process.

The Dirac theory would generate, in the restricted set of processes $q g \rightarrow \tilde{q}_{R} \tilde{g}_{D}^{c}$ and $\tilde{q}_{L} \tilde{g}_{D}$, the same degree of polarization.

The single polarization vector can be determined experimentally by using the two methods discussed in the previous section:

(a) Tagging of the $\tilde{u}_{L}, \tilde{d}_{L}^{*}$ or $\tilde{d}_{L}, \tilde{u}_{L}^{*}$ squarks by searching for $\ell^{+}$or $\ell^{-}$final states allows one to reconstruct the spin with analysis power $\kappa=\mid B R_{\tilde{d}}-$ $B R_{\tilde{u}} \mid /\left[B R_{\tilde{d}}+B R_{\tilde{d}}\right]$ in the first two generations.

(b) Analysis of top squark and sbottom final states allows the reconstruction of the spin vector, without dilution by destructive interference effects of different flavors but with some dilution due to the superposition of near and far-top quarks.

\section{B. Spin-phenomenology of the super-Compton process}

The spin effects in the super-Compton process are described by the polarization vectors $\mathcal{C}_{\mu}$ and $\mathcal{P}_{\mu}$ in the gluino production and decay processes [15]:

$$
d \sigma=d \sigma^{\mathrm{unpol}}\left[1-\mathcal{C}_{\mu} \mathcal{P}_{\mu}\right] .
$$

Depending on the sign of the product of the production and decay polarization vectors either constructive or destructive spin effects are generated, affecting, in principle, all experimental observables.

Along with the foregoing gluino-pair discussion we illustrate the spin phenomenology in the super-Compton process again by analyzing jet-jet invariant masses. The reference jet will be the primary squark decay jet $q_{3}$ recoiling against the gluino decay antiquark jet $\bar{q}_{2}$ and the secondary quark decay jet $q_{1}$ emitted in the squark decay of the gluino chain, cf. Fig. 7 :

$$
\tilde{q} \tilde{g} \rightarrow\left[\tilde{q}_{3}\right]\left[\bar{q}_{2} \tilde{q}_{2}\right] \rightarrow q_{3} \bar{q}_{2} q_{1} \tilde{\chi} \tilde{\chi} .
$$

The incoming quarks will be taken as $u, d$ valence quarks.

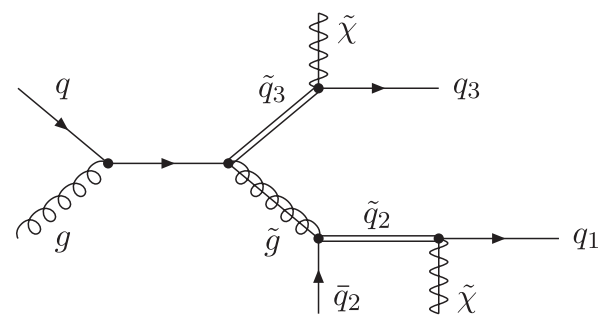

FIG. 7. Decay chains in the super-Compton process. While $q$ is effectively restricted to valence $u, d$ quarks, the squark $\tilde{q}_{2}$ may be substituted by the corresponding antisquark, the attached (anti)quarks correspondingly.

Three invariant masses $q_{1} \bar{q}_{2}, q_{1} q_{3}, \bar{q}_{2} q_{3}$ can be formed from the three final-state quark momenta. Since the polarization vector $\mathcal{P}$ can be varied by picking $L$ squarks or antisquarks in the $\tilde{g}$ decay state, different values are predicted for the jet-jet invariant masses.

Gluino Majorana theories generate equal- and oppositehelicity (anti)quark final states $\bar{q}_{2} q_{3}$ while Dirac theories restrict these final states to equal-helicity pairs.

The average values of the three jet-jet invariant masses for $\tilde{u}_{L} \tilde{u}_{L}$ and $\tilde{u}_{L} \tilde{u}_{L}^{*}$ final states coming with $u_{L}$ and $u_{L} / \bar{u}_{R}$ quark jets are presented in Table II, for identified jets in the upper row, and jet pairs ordered according to rising invariant masses $M_{\text {inv }}$ in the second row.

As evident from the table, the combination $\bar{q}_{2} q_{3}$, involving the primary antiquark decay jet of the polarized gluino, provides the highest sensitivity to spin effects. This combination is mapped, on the average, to the second largest invariant mass in the $M_{\text {inv }}$ ordered three-jet ensemble. A clear distinction emerges between constructive and destructive spin effects in this observable. If large invariant masses for the three-jet final states are selected, say $M_{j j j}>$ $2.5 \mathrm{TeV}$, the longitudinal gluino polarization is greatly enlarged, boosting forward or backward the recoiling de-

TABLE II. Invariant jet-jet masses in the super-Compton process. The average values $\left\langle M^{2}\right\rangle$ and the differences $\Delta M^{2}$ of the invariant mass distributions for $\tilde{u}_{L} \tilde{u}_{L}$ and $\tilde{u}_{L} \tilde{u}_{L}^{*}$ intermediate states are shown for identified jets (upper section) and for jets ordered according to invariant mass (middle section). The lower section shows the enhanced polarization effects if large invariant masses $M_{j j j}>2.5 \mathrm{TeV}$ are selected.

\begin{tabular}{lccc}
\hline \hline Super-Compton: $\tilde{q} \tilde{g} \rightarrow[\tilde{q}][\bar{q} \tilde{q}] \rightarrow$ & $q_{3} \bar{q}_{2} q_{1} \tilde{\chi} \tilde{\chi}$ & \\
Original quarks & $q_{1} \bar{q}_{2}$ & $q_{1} q_{3}$ & $\bar{q}_{2} q_{3}$ \\
\hline$\left\langle M^{2}\right\rangle\left[10^{4} \mathrm{GeV}^{2}\right]$ & 2.39 & 48.3 & 7.13 \\
$\Delta M^{2} /\left\langle M^{2}\right\rangle[\%]$ & $<0.1$ & 0.8 & 7.9 \\
$\mathrm{M}_{\text {inv }}$ ordered jets & small & medium & large \\
$\left\langle M^{2}\right\rangle\left[10^{4} \mathrm{GeV}^{2}\right]$ & 1.80 & 7.33 & 48.7 \\
$\Delta M^{2} /\left\langle M^{2}\right\rangle[\%]$ & 5.0 & 5.9 & 0.7 \\
$\mathrm{M}_{\mathrm{jij}}>2.5 \mathrm{TeV}:$ & small & medium & large \\
$\left\langle M^{2}\right\rangle\left[10^{4} \mathrm{GeV}^{2}\right]$ & 2.46 & 76.3 & 775 \\
$\Delta M^{2} /\left\langle M^{2}\right\rangle[\%]$ & 10.3 & 21.2 & 2.0 \\
\hline \hline
\end{tabular}




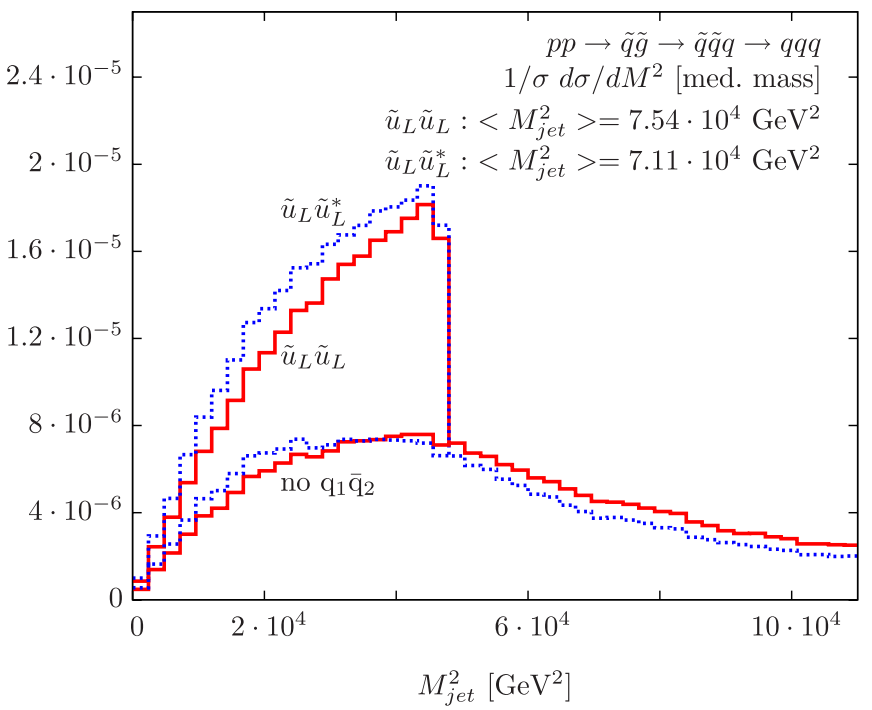

(a)

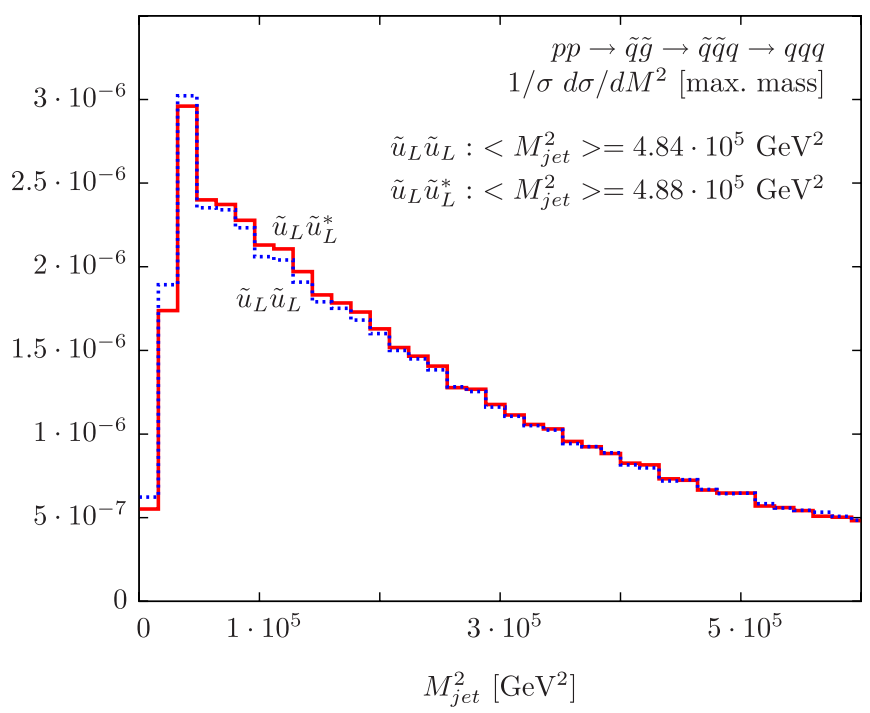

(b)

FIG. 8 (color online). Mass distribution of (a) the second largest invariant mass; the same-side $q_{1} \bar{q}_{2}$ parton combination is subtracted in the lower curves; (b) the distribution for jets of maximal invariant mass, corresponding largely to partons $q_{1} q_{3}$.

cay squark, and raising or lowering the jet-jet invariant masses accordingly. The spin-dependent distributions of the second largest invariant mass are depicted in Fig. 8(a). (The contamination due to same-side $\bar{q}_{2} q_{1}$ partons, which generate the wedge with the standard sharp edge at $\approx$ $\left[M_{\tilde{g}}^{2}-M_{\tilde{q}}^{2}\right]$ but do not give rise to spin asymmetries, is subtracted for illustration in the two lower curves.) Correlations among squark decay jets $\left[q_{1} q_{3}\right]$ are small, Fig. 8(b), as expected.

\section{SUMMARY}

Spin correlations in gluino-pair production and polarization effects in single gluino production of the superCompton process affect the distributions of the experimentally observed final states. In this report we have analyzed the theoretical basis of these effects, calculating the twogluino spin-correlation matrix in the first case and the gluino polarization vector in the second. A few examples for jet invariant masses illustrate the size of these effects at the theoretical level. They become relevant only if $L$ and $R$ squarks, coming in association with $R / L$ polarized antiquarks, etc., are discriminated by measuring, for instance, charges in the third generation. The spin effects are modest, typically of about $10 \%$ in the spin-sensitive observables. Nevertheless, when the LHC potential is fully exploited for precision measurements and analyses are performed to determine supersymmetry parameters like mixings, couplings, etc., such effects must be controlled properly in the analyses of the measured final states. The report is intended to provide a first step in this direction.

\section{ACKNOWLEDGMENTS}

We acknowledge helpful discussions with G. Polesello on experimental aspects of this study. P. M.Z. is grateful to the Institut für Theoretische Physik E for the warm hospitality extended to him at the RWTH Aachen.
[1] Yu. A. Golfand and E. P. Likhtman, JETP Lett. 13, 3214 (1971); J. Wess and B. Zumino, Nucl. Phys. B70, 39 (1974).

[2] H. P. Nilles, Phys. Rep. 110, 1 (1984); H. E. Haber and G. L. Kane, Phys. Rep. 117, 75 (1985).

[3] J. Wess and J. Bagger, Supersymmetry and Supergravity (Princeton University Press, Princeton, 1992) p. 259; P. Binetruy, Supersymmetry: Theory, Experiment and Cosmology (Oxford University Press, Oxford, 2006), p. 520; M. Drees, R. Godbole, and P. Roy, Theory and
Phenomenology of Sparticles: An Account of FourDimensional $N=1$ Supersymmetry in High Energy Physics (World Scientific, Hackensack, 2004) p. 555.

[4] S. Dawson, E. Eichten, and C. Quigg, Phys. Rev. D 31, 1581 (1985).

[5] W. Beenakker, R. Höpker, M. Spira, and P. M. Zerwas, Nucl. Phys. B492, 51 (1997).

[6] A. J. Barr, Phys. Lett. B 596, 205 (2004); J. M. Smillie and B. R. Webber, J. High Energy Phys. 10 (2005) 069; A. Alves, O. Eboli, and T. Plehn, Phys. Rev. D 74, 095010 
(2006); C. Csaki, J. Heinonen, and M. Perelstein, J. High Energy Phys. 10 (2007) 107.

[7] B. C. Allanach et al., in Proc. of the APS/DPF/DPB Summer Study on the Future of Particle Physics, Snowmass (Colorado), 2001 (unpublished).Eur. Phys. J. C 25, 113 (2002); J. A. Aguilar-Saavedra et al., Eur. Phys. J. C 46, 43 (2006).

[8] S. Y. Choi, M. Drees, A. Freitas, and P. M. Zerwas, Phys. Rev. D 78, 095007 (2008).

[9] M. M. Nojiri and M. Takeuchi, Phys. Rev. D 76, 015009 (2007).

[10] P. Fayet, Nucl. Phys. B113, 135 (1976); L. ÁlvarezGaumé and S. F. Hassan, Fortschr. Phys. 45, 159 (1997); M. M. Nojiri et al., arXiv:0802.3672.

[11] S. Y. Choi, M. Drees, J. Kalinowski, J. M. Kim, E. Popenda, and P. M. Zerwas, Phys. Lett. B 672, 246 (2009); T. Plehn and T. M. P. Tait, J. Phys. G 36, 075001 (2009).

[12] E. Popenda, Diploma thesis, Institut für Theoretische Physik, RWTH Aachen, 2009.

[13] S. Y. Choi, K. Hagiwara, Y. G. Kim, K. Mawatari, and P. M. Zerwas, Phys. Lett. B 648, 207 (2007).

[14] A. Kulesza and L. Motyka, Phys. Rev. Lett. 102, 111802 (2009).
[15] J. H. Kühn, A. Reiter, and P. M. Zerwas, Nucl. Phys. B272, 560 (1986).

[16] W. Bernreuther, A. Brandenburg, Z. G. Si, and P. Uwer, Nucl. Phys. B690, 81 (2004).

[17] W. Beenakker, R. Höpker, and P. M. Zerwas, Phys. Lett. B 378, 159 (1996).

[18] M. Mühlleitner, A. Djouadi, and Y. Mambrini, Comput. Phys. Commun. 168, 46 (2005).

[19] W. Beenakker, R. Hopker, T. Plehn, and P. M. Zerwas, Z. Phys. C 75, 349 (1997).

[20] A. Freitas, P.Z. Skands, M. Spira, and P. M. Zerwas, J. High Energy Phys. 07 (2007) 025.

[21] J. Pumplin, D. R. Stump, J. Huston, H. L. Lai, P. M. Nadolsky, and W. K. Tung, J. High Energy Phys. 07 (2002) 012.

[22] H. Murayama, I. Watanabe, and K. Hagiwara, Report No. KEK-91-11; T. Stelzer and W. F. Long, Comput. Phys. Commun. 81, 357 (1994); F. Maltoni and T. Stelzer, J. High Energy Phys. 02 (2003) 027; J. Alwall, P. Artoisenet, S. de Visscher, C. Duhr, R. Frederix, M. Herquet, and O. Mattelaer, AIP Conf. Proc. 1078, 84 (2009).

[23] W. Hollik, E. Mirabella, and M. K. Trenkel, J. High Energy Phys. 02 (2009) 002. 